\title{
Reliability analysis of a timber truss system subjected to decay
}

\author{
Ricardo D. Brites ${ }^{\mathrm{a}}$, Luís C. Neves ${ }^{\mathrm{b}}$, J. Saporiti Machado ${ }^{\mathrm{c}}$, Paulo B. Lourenço ${ }^{\mathrm{a}, *}$, \\ Hélder S. Sousa ${ }^{\mathrm{a}}$ \\ ${ }^{a}$ Universidade do Minho, ISISE, Dept. of Civil Engineering, Azurém P-4800-058 Guimarães, PORTUGAL \\ ${ }^{b}$ UNIC, Universidade Nova de Lisboa, Dept. of Civil Engineering, Quinta da Torre, 2829-516 Monte da \\ Caparica, PORTUGAL \\ ${ }^{c}$ Laboratório Nacional de Engenharia Civil, Núcleo de Estruturas de Madeira, Av. Do Brasil, 101 \\ 1700-066 Lisboa, PORTUGAL
}

\begin{abstract}
Assessing the safety of existing timber structures is of paramount importance for taking reliable decisions on repair actions and their extent. The results obtained through semi-probabilistic methods are unrealistic, as the partial safety factors present in codes are calibrated considering the uncertainty exhibited by new structures. In order to overcome these limitations, and also to include the effects of decay in the safety analysis, probabilistic methods, based on Monte-Carlo simulation are applied here to assess the safety of existing timber structures. In particular, the impact of decay on structural safety is analyzed and discussed using a simple structural model, similar to that used for current semi-probabilistic analysis.

Keywords: structural reliability, timber structures, biological deterioration, model, fungi, damage
\end{abstract}

\section{Introduction}

The safety evaluation of an existing structure is a key task if a decision to maintain it in service is to be taken. This task allows also the definition and prescription of conservation and strengthening measures, if needed. The process requires knowledge on the geometric and mechanical properties of timber members, as well as on the loads

\footnotetext{
${ }^{*}$ Corresponding author. Tel.:+351253510200; fax:+351253510217. E-mail address: pbl@ civil.uminho.pt
}

Preprint submitted to Elsevier

July 30, 2012 
that the structure was and will be subjected to. Although the geometrical properties can be gathered by direct in situ measurements, strength capacity and stiffness are more difficult to obtain.

Moreover, in old structures, the mechanical properties of timber are affected, not only by the duration of load and moisture content, but also by biological activity, which is common in elements in direct contact with masonry walls subjected to water infiltration. Due to the influence of different issues not considered in the original design, like ageing, load history, decay, etc., the usual procedure of design for new timber structures cannot be directly applied in the safety evaluation of ancient structures. The strict application of design codes to existing structures often leads to an inadequate safety evaluation and, often, unnecessary structural replacement. Most advanced safety evaluation models for timber structures, like the probabilistic safety evaluation, are seldom used due the difficulty in modeling important characteristics of wood, such as duration of load and influence of defects (knots, etc.). Recent publications and works in these areas have provided information that helps overcoming these issues (Köhler et al., 2007).

The influence of biological decay in structural safety can be critical to choose if, where and when a repair is needed, but there is no practical means to take biological decay into account in a semi-probabilistic analysis, since existing models relate the loss of strength with the loss of weight. A more consistent approach, relating the time of attack with the loss of mechanical properties is required for safety analysis purposes. The assumption made in the present paper is that when decay is present in a structural timber member, a reduced cross-section can be obtained. The same concept is applied to structural fire design of timber structures, in which the charred superficial layer is removed and the remaining cross-section is assumed to retain its full strength capacity. Here, the effect of initial fire degradation (with alteration of wood chemical properties and some cell wall damage) is compared with incipient fungi degradation.

Timber decay also has significant impact on the performance of timber joints. However, this aspect is not considered in the present paper, as no data on this impact, for the timber joints commonly used in historical structures in Europe, exists.

In the last decades, with the improvement of computational power and the developments in structural reliability, the application of probabilistic methods in ordinary 
structures became affordable. The development of structural codes with an explicit probabilistic approach, namely the Probabilistic Model Code (PMC) (JCSS, 2001) has allowed the spread of reliability-based methods in the safety evaluation of common structures.

Probabilistic methods for safety analysis are often based in the evaluation of a limit state function, which, in case of analysis of material degradation leading to failure of performance, requires an understanding of the structure environment, the transfer mechanism, the environmental action and leading to action effects that may result in failure. In this scope, the International Standard ISO 13823 (2008) specifies general principles and recommends procedures for the verification of the durability of structures subject to known or foreseeable environmental actions, including mechanical actions, causing material degradation performance reduction. In this case, for material deterioration resulting in failure due to loss of resistance, the ultimate limit state is defined when the resistance of the component or structure becomes equal to or less than the internal force. Therefore, structures and their components shall be designed, inspected, maintained and repaired in such a way that, under foreseeable environmental conditions, they maintain their required performance during their design lives with sufficient reliability for the safety and comfort of users and the intended use of the structure, thus providing that the service life of the structure meets or exceeds the design life.

\section{Simplified probabilistic modelling: the Monte Carlo method}

Probabilistic methods of safety analysis allow explicit consideration of uncertainty in the structural model, even if more computational power and a deeper knowledge of the modelled variables are needed. The Monte Carlo method, used hereafter, is a simple probabilistic method that evaluates the Limit State Function (LSF) in a predefined number of points randomly chosen. Basically, this is achieved by simulating a large number of events and evaluating the structural response for each one of them, being the obtained response statistically treated.

There are several methods within the Monte Carlo simulation, all of them based on 
pure simulation, which can be synthesized in the following steps:

1. Definition of the problem in terms of random variables;

2. Definition of the parameters of random variables (distribution, parameters and correlation among variables);

3. Simulation of $N_{\text {sim }}$ vectors of random variables;

4. "Deterministic" structural evaluation of each one of the $N_{\text {sim }}$ samples;

5. Defining $N_{f}$ as the number of simulations where the limit state function is violated, the probability of failure $p_{f}$ can be estimated by $\overline{p_{f}}$ :

$$
p_{f} \simeq \overline{p_{f}}=\frac{N_{f}}{N_{\text {sim }}}
$$

6. Definition of the accuracy and efficiency of the simulation, by means of varying $N_{\text {sim }}$ and evaluating how much it affects the results.

The number of simulations needed to keep the error below a predefined value is strongly dependent on the probability of failure. A practical way of estimating the number of simulations needed for values of probability of failure about $p_{f}=10^{-n}$ is to make at least $10^{n+2}$ simulations. Shooman (1968) proposed the expression for the maximum error in the estimate of $p_{f}$ for a $95 \%$ confidence interval, given by:

$$
\text { error }=2 \sqrt{\frac{1-p_{f}}{N_{\text {sim }} \cdot p_{f}}}
$$

\section{Probabilistic models for timber mechanical properties}

The adopted probabilistic models for the mechanical properties of timber are based on the theory described in PMC (Köhler et al., 2007, JCSS, 2001), which requires three reference variables, namely bending modulus of elasticity $(M O E)$, bending strength $\left(f_{m}\right)$ and density $(\rho)$. All the other relevant mechanical properties are established by empirical relationships with the reference variables. The proposed relations and statistical distributions are presented in Table 1.

Correlation among these properties must also be considered. A correlation matrix is defined empirically in JCSS (2001), where 0.8, 0.6, 0.4 and 0.2 indicate high, average, low and very low correlation, respectively, see Table 2. 
Table 1: Density probability function of timber mechanical properties, defined as a function of the reference variables (in grey rows), according to JCSS (2001)

\begin{tabular}{lccc} 
Property, $X$ & Distribution & Expected value $E[X]$ & $\begin{array}{c}\text { Coefficient of } \\
\text { variation } \operatorname{CoV}[X]\end{array}$ \\
\hline Bending strength, $f_{m}$ & Lognormal & $E\left[f_{m}\right]$ & 0.25 \\
Bending MOE, $E_{m}$ & Lognormal & $E\left[E_{m}\right]$ & 0.13 \\
Density, $\rho_{m}$ & Normal & $E\left[\rho_{m}\right]$ & 0.10 \\
Tensile strength parallel to the grain, $f_{t, 0}$ & Log-normal & $0.6 E\left[f_{m}\right]$ & $1.2 \operatorname{CoV}\left[f_{m}\right]$ \\
Tensile strength perpendicular to the grain, $f_{t, 90}$ & Weibull & $0.015 E\left[\rho_{m}\right]$ & $2.5 \operatorname{CoV}\left[\rho_{m}\right]$ \\
MOE in tension parallel to the grain, $E_{t, 0}$ & Log-normal & $E\left[E_{m}\right]$ & $\operatorname{CoV}\left[E_{m}\right]$ \\
MOE in tension perpendicular to the grain, $E_{t, 90}$ & Log-normal & $E\left[E_{m}\right] / 30$ & $\operatorname{CoV}\left[E_{m}\right]$ \\
Compression strength parallel to the grain, $f_{c, 0}$ & Log-normal & $5 E\left[f_{m}\right]^{0.45}$ & $0.8 \operatorname{CoV}\left[f_{m}\right]$ \\
Compression strength perpendicular to the grain, $f_{c, 90}$ & Normal & $0.008 E\left[\rho_{m}\right]^{0.45}$ & $\operatorname{CoV}\left[\rho_{m}\right]$ \\
Shear modulus, $G_{v}$ & Log-normal & $E\left[E_{m}\right] / 16$ & $\operatorname{CoV}\left[E_{m}\right]$ \\
Shear strength, $f_{v}$ & Log-normal & $0.3 E\left[f_{m}\right]^{0.8}$ & $\operatorname{CoV}\left[f_{m}\right]$
\end{tabular}

Table 2: Correlation matrix for material properties, according to JCSS (2001)

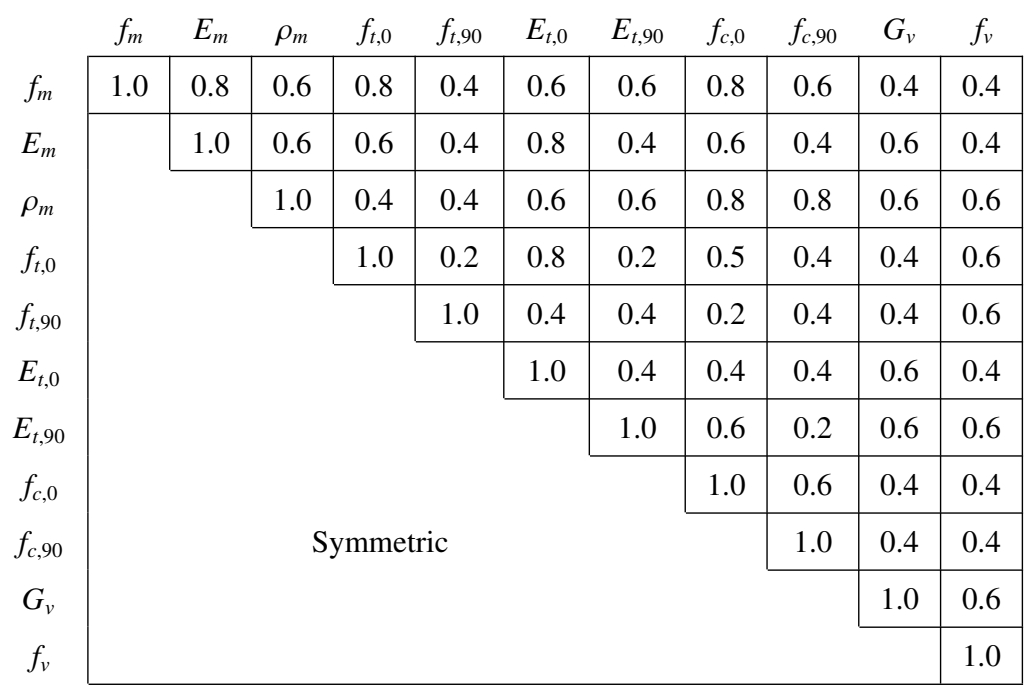




\section{Probabilistic models for loads}

Loads can be distinguished between dead loads and live loads. The first group includes loads that are always present in the structure and that have values approximately constant over time. These actions are the most relevant for long term effects (creep), being associated with the weight of the structure and the supported elements (tiles, ceilings, etc.). Live loads are stochastic processes in time.

The probabilistic modelling of dead loads can be easily achieved by considering the randomness of cross-sections and density of the materials. Live loads are more complex to model and only simplified extreme value distributions are considered here. It is noted that snow and wind loads are often critical for the design of timber roofs, while imposed loads are often critical for the design of timber floors.

\section{Limit state functions and reference values for the reliability index}

Limit State Functions (LSF) are used to determine if a given element/structure continues to fulfil its function as desired. These functions should be defined in such way that they assume negative values if violated. The simplest way of expressing limit state functions is:

$$
G(x)=R(x)-S(x) \text { or } \quad G(x)=1-\frac{S(x)}{R(x)}
$$

Here, $R(x)$ represents the mechanical resistant properties and $S(x)$ the effects of applied actions. The safety check of timber structures for the Ultimate Limit Sates (ULS) requires that several types of internal forces are evaluated in the same function. For these cases, a more complex formulation for the limit state function is required.

Köhler et al. (2007) suggest the adjustment of the Eurocode 5 (EN 1995:1-1, 2004) formulation by removing the partial safety factor $\gamma_{M}$ of this formulation and making the equation negative if the safety check is not fulfilled. As an example, it is possible to consider EN 1995:1-1 (2004), for verification of columns subjected to either compression or combined compression and bending 4 . The Eurocode 5 formulation is:

$$
\frac{\sigma_{c, 0, d}}{k_{c, y} f_{c, 0, d}}+\frac{\sigma_{m, y, d}}{f_{m, y, d}}+k_{m} \frac{\sigma_{m, z, d}}{f_{m, z, d}} \leq 1
$$


Here, $\sigma_{c, 0, d}, \sigma_{m, y, d}$ and $\sigma_{m, z, d}$ are the design compressive stress along the grain, design bending stress about the principal y-axis and design bending stress about the principal z-axis (respectively); $f_{c, 0, d}, f_{m, y, d}$ and $f_{m, z, d}$ are the design compressive strength along the grain, design bending strength about the principal y-axis and design bending strength about the principal z-axis (respectively); $k_{c, y}$ is the instability factor and $k_{m}$ a factor that considers the re-distribution of bending stresses in a cross-section (EN 1995:11, 2004). In the LSF formulation, this equation should be expressed as:

$$
G(x)=1-\left[\frac{\sigma_{c, 0}}{k_{c, y} f_{c, 0}}+\frac{\sigma_{m, y}}{f_{m, y}}+k_{m} \frac{\sigma_{m, z}}{f_{m, z}}\right]
$$

The absence of the $d$ in the subscript of variables stresses that this verification is now made with the simulated values, instead of design values. The safety condition of the structure is now established by limiting the probability of failure to a given value.

Another common way of expressing the same result is by using the reliability index $\beta$, which can be calculated directly from the probability of failure by $\beta=-\Phi^{-1}\left(p_{f}\right)$, where $\Phi^{-1}$ denotes the inverse of the standard normal distribution. Target values for this parameter are established as a function of the importance of the building and the cost to upgrade its safety. Hence, critical structures, like hospitals and bridges, must exhibit a higher reliability index than ordinary structures, which implies that the probability of failure should be (much) smaller. The values suggested in JCSS (2001) are given in Table 3. In new ordinary structures a target reliability index of $\beta=4.2$ is usually used, but in existing structures the cost of structural interventions is generally high and a lower target reliability index of $\beta=3.3$ is used instead. This is the value assumed hereafter.

\section{Probabilistic models for fungal decay in timber structures}

Degradation of timber structures often occurs with the decay of elements in direct contact with masonry walls, where the high moisture content and mild temperatures provides an optimal media for the development of fungi.

Leicester (2001) refers that after the mycelia mat is established, temperature and moisture content are the most important variables that control the rate of fungi growth. 
Table 3: Target reliability indexes $\beta$ suggested by JCSS (2001), established as a function of consequences of collapse and difficulty to upgrade the safety level

\begin{tabular}{cccc} 
Cost for upgrading & \multicolumn{3}{c}{ Collapse consequences } \\
the safety level & Low & Medium & High \\
\hline High & $\beta=3.1$ & $\beta=3.3$ & $\beta=3.7$ \\
Average & $\beta=3.7$ & $\beta=4.2$ & $\beta=4.4$ \\
Low & $\beta=4.2$ & $\beta=4.4$ & $\beta=4.7$ \\
\hline
\end{tabular}

Temperatures in the range $5^{\circ} \mathrm{C}-30^{\circ} \mathrm{C}$ are adequate for fungi growth, being fungi latent below $5^{\circ} \mathrm{C}$ and killed above $65^{\circ} \mathrm{C}$. In terms of moisture content, values below the fibre saturation point or above $80 \%$ of cell cavity filled with water are inadequate. Idealised relations between temperature and rate of growth, and moisture content and rate of growth, are given in Figure 1.

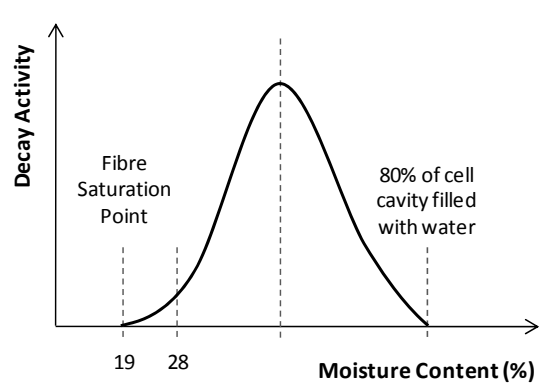

(a)

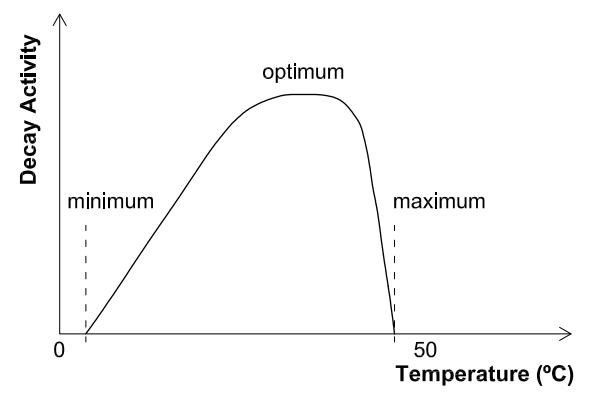

(b)

Figure 1: Theoretical fungal growth as a function of (a) moisture content and (b) temperature, according to Zabel and Morrell (1992)

Recent works from Wang et al. (2008) and Leicester et al. (2009) allow the definition of an empirical model that defines the depth of fungal attack as a function of time of exposure, for both in and above-ground decay. In this model, after an initial incubation period in which there is no visible progression, the decay rate is roughly constant over time, see Figure 2. The decay rate per unit of time (mm/year), denoted as $r$, can be established using two parameters (dimensionless parameters calibrated to give $r$ in mm/year), which depend on the climate and on a coefficient that models the 
durability and structural conditions of the timber element, according to Equation 6:

$$
r=k_{0} \cdot k_{\text {climate }}
$$

The coefficient $k_{0}$ is given by the consideration of several parameters, such as $k_{\text {wood }}$ (wood parameter), $k_{t}$ (thickness parameter), $k_{w}$ (width parameter), $k_{n}$ (connector parameter), $k_{g}$ (geometry parameter) and $k_{p}$ (paint parameter), according to Equation 7:

$$
k_{0}=k_{\text {wood }} \cdot k_{t} \cdot k_{w} \cdot k_{n} \cdot k_{g} \cdot k_{p}
$$

The paramenter $k_{\text {wood }}$ depends on the timber durability class as defined in AS 56042005 (AS 5604-2005, 2005) and $k_{\text {climate }}$ depends on climate factors. The $k_{\text {climate }}$ is a parameter that considers the effect of climate conditions with respect to the annual time of rainfall, $t_{\text {rain }}$, in hours. The $k_{\text {climate }}$ is given according to Equation 8, and a correlation coefficient between $k_{\text {climate }}$ and $t_{\text {rain }}$ of 0.44 ,is considered (Wang and Leicester, 2008). According to the same authors, regarding the influence of temperature on decay rate, no noticeable correlation was found.

$$
k_{\text {climate }}=0.03 \cdot t_{\text {rain }}^{0.5}
$$

Since $k_{\text {wood }}$ depends on the durability of the wood species with respect to the pathological agents and $k_{\text {climate }}$ depends on the vulnerability to fungal decay due to climatic variation in a given location, these parameters were considered uncorrelated. Lognormal distributions are introduced according to the data from field tests in (Wang and Leicester, 2008). The parameters $k_{\text {wood }}$ and $k_{\text {climate }}$ have been calibrated for Australian species and climate, with the values presented in Table 4 for above-ground timber elements (Leicester et al., 2009). Here, four climatic hazard zones are considered (A to D), where hazard zone A corresponds to the climate with lower annual time of rainfall and hazard zone to the climate with higher annual time of rainfall (see Table 4 and Table 5).

The thickness parameter $k_{t}$ and the width parameter $k_{w}$ take into account the effects of drying restraint in the transverse direction to timber grain and to the cross-grain direction, respectively, depending on the thickness and width of the member and if the 
element is in contact with other elements. The parameter $k_{t}$ takes values between 0.5 and 1 for a thickness of the timber element between 10 and $20 \mathrm{~mm}$, respectively. The parameter $k_{w}$ takes values between 1 and 2 for a width of the timber element between 50 and $200 \mathrm{~mm}$, respectively. For $k_{t}$ and $k_{w}$, intermediate values are calculated by linear interpolation. The connector parameter $k_{n}$ takes into consideration the effect of the presence of a connector on the decay surface, taking a value of 1 when there is no connector and a value of 2 when a connector is present. The geometry parameter $k_{g}$ is calculated by the multiplication of a contact factor $k_{g 1}$ that depends on the type of contact between members (flat, embedded or non-contact) and a position factor $k_{g 2}$ that depends on the orientation of the decay face. The paint parameter $k_{p}$ takes into account the effect of paint, having values of 1 for unpainted timber and values between 1.3 and 3.5 (depending on the timber class) for painted timber.

Table 4: Suggested parameters for $k_{\text {climate }}$ distribution, according to Leicester et al. (2009)
\begin{tabular}{cc} 
Zone & Mean $\left(k_{\text {climate }}\right)$ \\
\hline A & 0.40 \\
B & 0.50 \\
C & 0.65 \\
D & 0.75 \\
\hline
\end{tabular}

Table 5: Suggested parameters for $k_{\text {wood }}$ distribution, according to Leicester et al. (2009)

\begin{tabular}{ccccc} 
& \multicolumn{4}{c}{ Mean $\left(k_{\text {wood }}\right)$} \\
\cline { 2 - 5 } & outer heartwood & inner heartwood & untreated sapwood & treated sapwood \\
\hline 1 & 0.50 & 1.00 & 6.52 & 0.25 \\
2 & 0.65 & 1.30 & 6.52 & 0.25 \\
3 & 1.15 & 2.30 & 6.52 & 0.25 \\
4 & 2.20 & 4.40 & 6.52 & 0.25 \\
\hline
\end{tabular}

Assuming that $k_{\text {climate }}$ and $k_{0}$ follow a lognormal distribution, it can be shown that $r$ is also lognormal, with an expected value $E[r]$ given by: 


$$
E[r]=E\left[k_{0}\right] \cdot E\left[k_{\text {climate }}\right]
$$

Another parameter necessary for the definition of decay is the time of incubation $t_{\text {lag }}$, which is the time between the installation of the timber element and the initiation of performance loss due to decay. This parameter, $t_{l a g}$ (years) can be estimated as a function of the decay rate $r$ (mm/year) according to experimental data (Leicester et al., 2009):

$$
t_{\text {lag }}=8.5 r^{-0.85}
$$

A lognormal distribution with an expected value given by Equation 10 can be used.

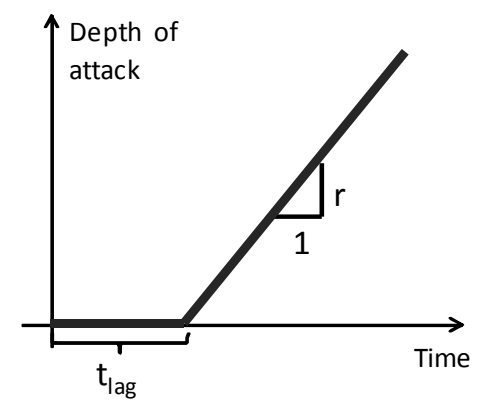

Figure 2: Numerical modelling of decay depth as a function of time: theoretical model Leicester et al. (2009)

Numerically, the depth of attack $d(\mathrm{~mm})$ over time can be modelled according to Equation 11:

$$
d(t)= \begin{cases}0 & t \leq t_{\text {lag }} \\ r \cdot\left(t-t_{\text {lag }}\right) & t>t_{\text {lag }}\end{cases}
$$

The calibration of the above model should be made for each wood species and fungus, and hence experimental tests are needed for engineering applications on different locations. For the case of protected structures, such as the case of sheltered elements of a roof system, the information about the climatic conditions of the surrounding environment should be accompanied by detailing the construction characteristics in order to define the mechanism through which the timber elements are put in contact with free 
moisture. The duration of this phenomenon is also important since the mould growth rate on a surface at a given time may be taken as a function of the cumulative sum of the periods that allowed for the growth of mould fungi (Viitanen, 1994). In protected structures, the free moisture may be consequence of rainwater ingress due to poor building practices or deficient maintenance, leakage from indoor sources or condensation. In the work of Foliente et al. (2002) it is mentioned that in order to assess the decay attack to sheltered timber members it is necessary to define the microclimate within the building, which may be related to the external climate, although the author is also mentioning that, in the absence of other information, the decay models developed for exposed construction appear to be a useful starting point. Environmental conditions such as the period of rainfall combined with the characterization of wind values (direction and magnitude) are directly connected with the possibility of leakage, as found in the CMHC report for Alberta, Canada, dwellings (Canada Mortgage and Housing Corporation, 2000), where it was concluded that buildings exposed to high wind conditions required upgraded construction practices and materials to prevent costly repairs for moisture-related damage. Further information about the relation between rainfall and wind is given in (Canada Mortgage and Housing Corporation, 2007). Although the effects of rainfall and wind intensity caused considerable moisture-related damages in Alberta dwellings, the consequences of the environmental conditions were significantly severer in the former study in British Columbia, Canada, where, due to poor design and detailing, substantial amounts of water would remain confined within the walls without proper ventilation and evaporation, leading to the decay of the timber elements. In that case, about $90 \%$ of the problems were thought to be related to interface details between wall components or at roof penetrations (Canada Mortgage and Housing Corporation, 1996). The failure of the building envelope by leakage, also known as the leaky buildings syndrome, results in the building's timber framing to remain wet for a considerable period of time, raising its moisture content and therefore allowing for fungal growth. Therefore, the detailing of construction and respective possibility of leakage are necessarily taken in consideration when assessing the decay in timber elements protected by the building's envelope. 


\section{Experimental characterization of an European wood specie subjected to decay}

For calibration of the previous model to the Portuguese conditions, a series of decay tests using Maritime Pine timber (Pinuspinaster Ait.) subjected to a brown rot fungus is under study in the Portuguese National Laboratory of Civil Engineering (LNEC), for different exposure periods and cross sections (Brites, 2011). Decay stages (from incipient decay to advanced decay), defined to each sample attending to visual inspection, and the time necessary to achieve each stage are recorded. Decay is being induced by placing the specimens in contact with a brown rot fungus, namely Coniophoraputeana (Shumach.) P. Karst., obtained in a laboratory growth culture. With an average periodicity of two months the specimens are being assessed visually and by their superficial hardness. The specimens were fully placed in contact with the laboratory culture and water was periodically added in order to obtain favorable conditions for fungal growth. After removal from the decay laboratory culture, the specimens' moisture content was measured. The smaller specimens presented a mean moisture content of $52.7 \%$ while, for the medium size specimens, a mean moisture content of $26.44 \%$ was found. At this stage, only the results considering the reference stage and the incipient stage for small $\left(20 \times 40 \times 120 \mathrm{~mm}^{3}\right)$ and medium size $\left(30 \times 90 \times 180 \mathrm{~mm}^{3}\right)$ specimens are available, since the exposure time so far (four years) was not sufficient to reach more severe decay stages. For these specimens the exposure time that lead to the consideration of an incipient decay stage is shown in Table 6, showing a $\mathrm{CoV}$ above $40 \%$.

Table 6: Statistic parameters for the exposure time needed to reach an incipient decay stag
\begin{tabular}{cccccc} 
Specimen & $\mu$ [days] & $\sigma$ [days] & CoV [\%] & No of specimens $^{\circ}$ \\
\hline $20 \times 40 \times 120$ & 259 & 114 & 44.0 & 45 \\
$30 \times 90 \times 180$ & 296 & 120 & 40.5 & 17 \\
\hline
\end{tabular}

\section{Influence of decay in timber connections}

In wood structures, connections between members are generally the most important source of ductility (Chui and $\mathrm{Ni}, 1998$ ), but may present as potential weak sections if deteriorated. The seasonal movement within members connected by traditional 
joints, such as mortise and tenon joints, often produces gaps between members leading to the presence and concentration of water leading to higher moisture contents and thus favorable conditions for biological decay growth. Design codes, such as Eurocode 5 (EN 1995:1-1, 2004), often present rules for load carrying capacity determination and detailing of mechanically jointed elements and connections with metal fasteners leaving a considerable lack of information in respect to traditional joints generally found in historical constructions. Moreover, the reviewed literature fails to address the effect of biological decay on the mechanical performance of traditional joints. Norms EN 330 (1993) and ENV 12037 (1996) present methodologies for field tests respectively in Ljoint and lap-joint connections regarding the effectiveness of wood preservatives but are still dependant of a visual rating scale. The period of time necessary to detect incipient decay in these types of joints was studied in Clausen et al. (2006), where decay was detected in lap-joints earlier than in L-joints. In the same work, it is also mentioned that the relationship between test conditions and initiation of decay, is still poorly understood and that the limitations of current aboveground test procedures include the subjective nature of the rating system and lack of accurate methods for detecting and measuring the extent of decay. With respect to mechanical connections, shear performance of the joints frequently controls the efficiency of the system and the performance of the structure and may be severely affected by the timber decay process. For example, the influence of decay in nailed connections was reported in Kent et al. (2005) where the cumulative energy dissipation was the most sensitive property to decay damage. Also the type of failure mode was dependent on the level of decay found. Inn Sawata et al. (2008) the shear performance of dowel-type timber joints affected by brown rot fungus was investigated and was concluded that the initial stiffness, yield load and maximum load of dowel-type joints were significantly reduced, even with a small wood mass loss, especially related to the decay around the dowel lead hole. In this work, the reduction of load carrying capacity due to decay is considered in terms of section properties loss. The failure of connections has not been considered, since structural models for deteriorated joints, commonly used in historical structures in Europe, should be defined and experimentally tested before their use in structural assessment. 


\section{Probabilistic evaluation of a traditional roof truss}

The methodology detailed above is now applied to a traditional roof truss, namely the $6.00 \mathrm{~m}$ span king post truss built in Maritime Pine wood shown in Figure 3.

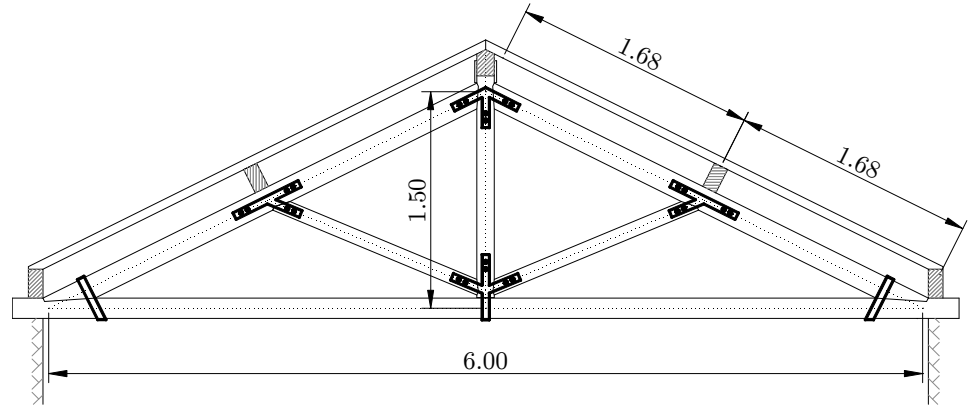

(a)

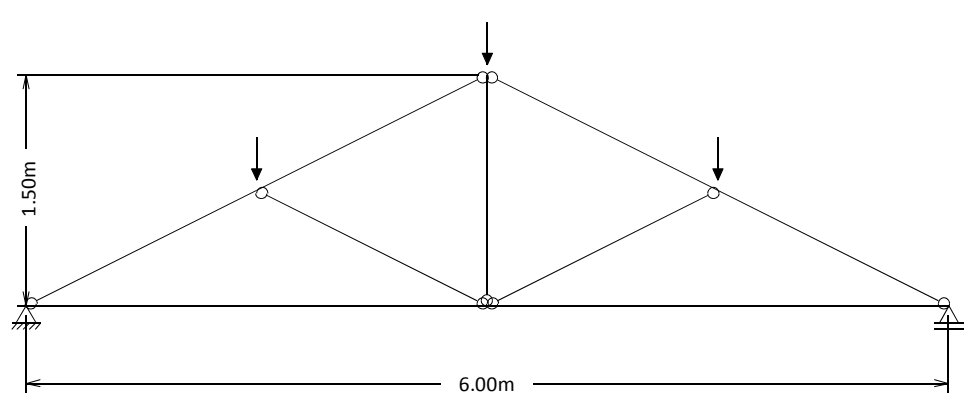

(b)

Figure 3: Roof truss studied: (a) "real" structure; (b) adopted truss model

The adopted random variables are given in Table 7, being the remaining mechanical properties established by the empirical relations given in Table 1, with the correlation matrix for mechanical properties defined in Table 2. The assumed crosssections replicate usual conditions and dead loads are established according to Eurocode 1 (EN 1991:1-3, 2003). The modification factor takes into account the duration of load effect and moisture content and its value is considered as constant and equal to $k_{\text {mod }}=0.60$, as described in EN 1995:1-1 (2004).

The variable load due to snow is calculated by the simplified method proposed by Ranta-Maunus (2004), where, in absence of more information, a $\mathrm{CoV}=0.40$ is 
recommended. A Gamma distribution was used for definition of the snow load (JCSS, 2001). Using the annual maximum values it is possible to define characteristic values of snow load with respect to a certain exceedance, directly associated to a certain mean recurrence interval. The characteristic value for snow load, in Eurocodes and National annexes, is the value corresponding to a probability of exceedance of $2 \%$ per year or a mean recurrence time of 50 years. The Gamma distribution is a two-parameter family of continuous probability distributions with a scale parameter $\theta$ and a shape parameter $k$. The two parameters for definition of the probability distribution may be found by:

$$
\begin{gathered}
\theta=\left|(E \cdot C o V)^{2} / E\right| \\
k=|E / \theta|
\end{gathered}
$$

The mean value for snow load at ground level was established by the method proposed in EN 1991:1-3 (2003). The snow shape coefficients $\left(\mu_{1}, \mu_{2}\right)$ vary with the roof pitch angle, being considered as $\mu_{1}=0.8$ and $\mu_{2}=1.6$, assuming the larger value in the left side. The non-symmetric load distribution is more stringent than the symmetric load distribution, being the difference larger as decay increases.

The decay rate for the rafters was determined by Equation 6, for a temperate climate $\left(k_{\text {climate }}=0.50\right)$ and assuming that the timber used is outer heartwood with durability class $3\left(k_{\text {wood }}=1.15\right)$. Considering the rafter cross section dimension, the effects of drying restraints are given by $k_{t}=1$ and $k_{w}=1.47$. Since the value of $k_{g}$ is dependent of the orientation of the decay face, different values were obtained, namely $k_{g}=0.9$ for the top face, $k_{g}=0.45$ for the bottom face and $k_{g}=0.3$ for the lateral faces. In all faces a non-contact situation was assumed. Also it was considered that no connector is present $\left(k_{n}=1\right)$ and that the timber element is unpainted $\left(k_{p}=1\right)$. All the adopted parameters and distributions are given in Table 7.

The computational routines needed to execute a Monte Carlo simulation were implemented in MATLAB, considering a linear elastic behaviour for the materials properties, a fragile structural behaviour and using the random number generators existing within the software. A flowchart of the solution is presented in Figure 4. A frag- 
Table 7: Adopted distributions and parameters for the random variables of the problem

\begin{tabular}{lccc}
\hline Property, $X$ & Distribution & $E[X]$ & $C o V[X]$ \\
\hline Bending strength, $f_{m}[\mathrm{MPa}]$ & Lognormal & 25.0 & 0.25 \\
Bending young modulus, $E_{m}[\mathrm{GPa}]$ & Lognormal & 15.0 & 0.13 \\
Timber density, $\rho_{m}\left[\mathrm{~kg} / \mathrm{m}^{3}\right]$ & Normal & 580 & 0.10 \\
Poisson coefficient, $v[-]$ & Deterministic & 0.30 & - \\
Tie beam cross section, $b \times h[\mathrm{~m}]$ & Normal & $0.12 \times 0.18$ & $0.10 \times 0.10$ \\
Principal rafter cross section, $b \times h[\mathrm{~m}]$ & Normal & $0.12 \times 0.18$ & $0.10 \times 0.10$ \\
King post and struts cross section, $b \times h[\mathrm{~m}]$ & Normal & $0.12 \times 0.12$ & $0.10 \times 0.10$ \\
Dead load, $G[k N]$ & Normal & 6.0 & 0.10 \\
Snow load (at ground level), yearly maxima, $S_{g}[\mathrm{kN}]$ & Gamma & 2.5 & 0.40 \\
Lateral side fungal decay rate, $r_{L}[$ mm/year $]$ & Lognormal & 0.253 & $0.70 ; 1.07 ; 1.60$ \\
Top side fungal decay rate, $r_{T}[\mathrm{~mm} /$ year $]$ & Lognormal & 0.759 & $0.70 ; 1.07 ; 1.60$ \\
Bottom side fungal decay rate, $r_{B}[\mathrm{~mm} /$ year $]$ & Lognormal & 0.380 & $0.70 ; 1.07 ; 1.60$ \\
Fungal incubation period, $t_{\text {lag }}[$ year $]$ & Lognormal & Eq. 10 & 0.40 \\
\hline
\end{tabular}

ile structural behaviour implies that the structure collapses when its first element collapses, which is generally true for simple roof trusses but false for complex structural solutions, as the latter can allow multiple load paths.

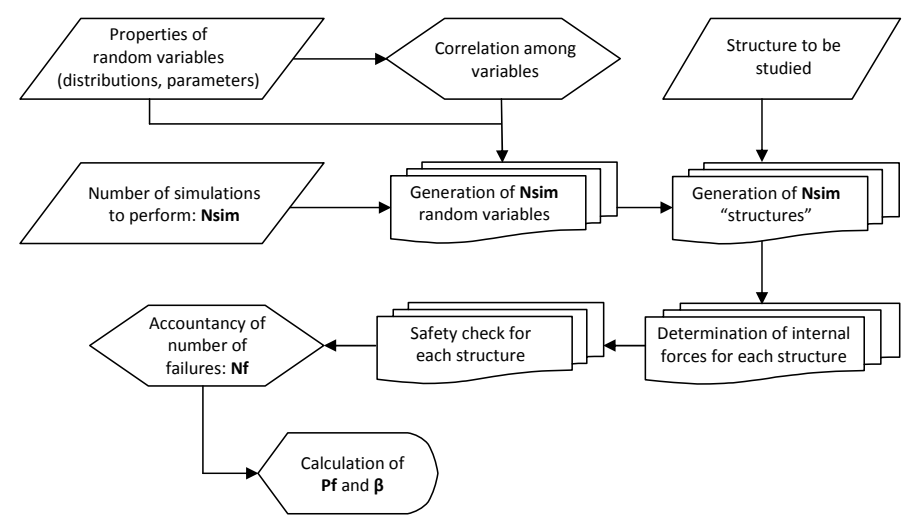

Figure 4: Flowchart of the Monte Carlo simulation implemented in MATLAB

The decay was introduced only in the lower part of the left rafter, and it was as- 
sumed as acting in the four sides of the element (Figure 5). This element was selected due its proximity to the support area and the bottom end of the roof, meaning that it is likely most often subjected to higher moisture contents, the main factor leading to decay in real timber structures. The decay rate $r$ was defined for each side of the element, thus the decay rate may not be constant between different sides. Since the decay rate must be calibrated to each case study and its variation may be very different, for the $\mathrm{CoV}$ of that parameter, a range of values between 0.70 and 2.00 was analysed, considering the works of Wang et al. (2008), Wang and Leicester (2008) and Leicester et al. (2009). The $C o V$ for $t_{l a g}$ was considered to be $40 \%$ based on the results found for the initiation of incipient decay in Brites (2011).

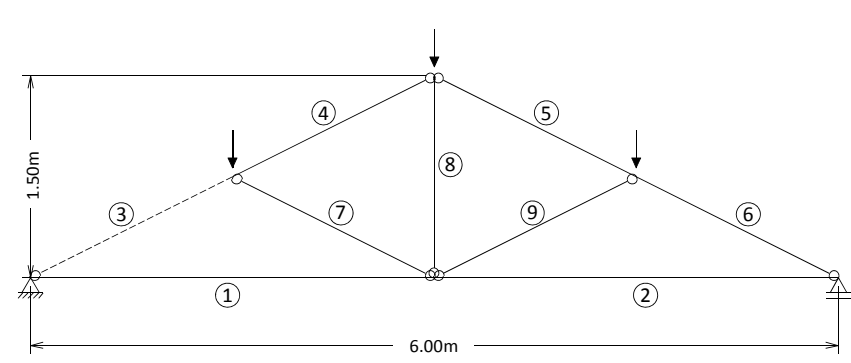

(a)

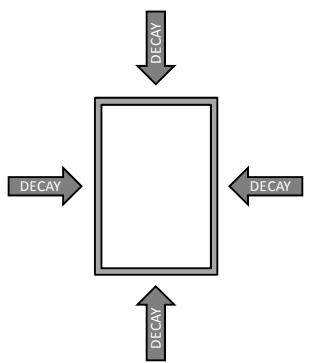

(b)

Figure 5: Modelling decay as a reduction of cross section: (a) decayed element (dashed line) and element identification; (b) cross section sides subjected to decay (all four sides)

A total of $1.2 \times 10^{7}$ simulations over a 50 year period was analyzed, leading to a probability of failure in absence of decay of $p_{f}=1.64 \times 10^{-7}(\beta=5.11)$. The reliability of the structure in the first years is conditioned by element 1 and 2 (tie beam), but after the decay starts the reliability of the structure becomes dependent of element 3 (left rafter). In this case, the reliability of the structure is highly dependent of the $C o V$ of the decay rate, having a steeper decrease of reliability for higher values of $C o V$. In the case of $C o V=2.00$, the probability of failure increases exponentially with decay, reaching $p_{f}=6.79 \times 10^{-2}(\beta=1.49)$ after 50 years of continuous decay. The evolution of the reliability index and of the probability of failure over time for the different CoVs of the decay rate is presented in Figure 6, where it can be seen that, when the 
decay starts to influence the reliability of the structure, the global probability of failure increases almost exponentially. As decay evolves, the reduction of the cross section in the affected element (element 3 ) leads to higher stress levels in adjacent elements. This behavior, with progressive increase of probability of failure in the sound elements nearby the decayed elements, is also observed in real structures, demonstrating that the model is capable of detecting the internal equilibrium rearrangements that the structure suffers due to decay.

It is noted that, for the adopted structure and an age of 50 years all models presented a $\beta<2.4$, which is clearly unsatisfactory regarding observations of real structures. Since this model contemplates a continuous decay process without any maintenance intervention, the probability of failure steadily increases along time. It is often observed that old timber roof trusses were submitted to repair or strengthening interventions which allows the structure to maintain a suitable safety level for a higher lifetime. On the other hand, the model proposed in Leicester et al. (2009) has been developed for exposed structures and thus it is likely that the assumed decay rates are too high for large sheltered timber elements and are over-conservative. Nevertheless, due to the inexistence of a sufficient large database for safety assessement of existing sheltered constructions of this type of timber species, the model has been adjusted regarding the safety level observed in real structures. A reduction factor was applied for the decay rate, in order to consider the lower period of time to which a shelterd timber structure is subjected, compared to an exposed structure. The application of this reduction factor also intends to calibrate the environmental action regarding the action effects and durability observed in real structures, by reverse use of the limit state method for durability proposed in ISO 13823 (2008). Assuming a target reliability level of $\beta=3.3$, structural repair or strengthening of the structure should be considered in year 14, 23 and 32 for the models where $C o V$ of $r$ equal to 2.00, 1.07 and 0.70, respectively. Structural interventions on this type of structures are not commonly observed in such early stage of the structure's life, therefore the decay model must be calibrated in order to simulate a higher structural life expectance. For that reason, the mean value of the decay rate was adjusted so that the structure would present a target reliability level of $\beta_{\text {target }}=3.3$ after 50 years. While reducing the decay rate it was considered that the $t_{\text {lag }}$ parameter should 
be maintained equal to the original analysis. Within the range of studied $C o V$ for decay depth and for the considered target reliability level it was found that a high $\mathrm{CoV}$ would lead to high reductions of the decay rate, inconsistent with the level of decay found in sheltered structures of this timber specie. Considering the lower variation of decay depth found in existing sheltered timber structures compared to exposed ones, a $\mathrm{CoV}$ of $r$ equal to 1.07 was found adequate for the model calibration in safety analysis. After an iterative procedure, a reduction factor of 3.2 was found suitable for the decay rate on each face of the element resulting in $r_{L}=0.079 \mathrm{~mm} /$ year; $r_{T}=0.237 \mathrm{~mm} /$ year and $r_{B}=0.119 \mathrm{~mm} /$ year. The evolution of the reliability index for the calibrated model and comparison with the original model is presented in Figure 7. The calibrated model now indicates that the decayed element will only start to influence the global reliability level roughly around year 16.

\section{Conclusions}

A simplified probabilistic model for the analysis of timber structures was implemented, assuming as random variables the cross-section, material properties and loads. The reliability concepts were then applied to a traditional king post truss, leading to the reliability index for a situation of no decay (time $=0$ ), as well as its evolution over time due the effect of decay (for a period of 50 years). The decay was modeled as a reduction of the cross section, being this parameter also considered as a random variable. It was found that the coefficient of variation for the decay rate largely influences the estimation of the reliability evolution through time. It was shown that when decay starts (after the incubation period) the probability of failure rises exponentially. Also, it was possible to calibrate the decay model parameters to the study case by considering a target reliability level that seems reasonable according to the observations in real structures. For this case, decay rates of $r_{L}=0.079 \mathrm{~mm} /$ year; $r_{T}=0.237 \mathrm{~mm} /$ year and $r_{B}=0.119 \mathrm{~mm} /$ year were found adequate, where the subscript $\mathrm{L}$ indicates lateral face, $\mathrm{T}$ indicates top face and $\mathrm{B}$ indicates bottom face.

Due to the limitations of the implemented model to take into account fundamental parameters like the effect of the duration of load (DOL) in the mechanical properties, 
some precaution is needed in the analysis of the obtained results. The inclusion of the DOL effects, as well as retrofitting actions and periodical inspections into the reliability index, would allow to compare the effect of different actions and to define the optimal timing to carry out the intervention for a given structure.

\section{Acknowledgements}

This work is funded by the Portuguese Foundation for Science and Technology (FCT) by the doctoral grant reference SFRH/BD/27353/06, as well as FCT Project PTDC/ECM/66527/2006 - Safety assessment of the safety of timber structures using non-destructive testing and stochastic analysis. The fifth author also acknowledges the financial support of FCT, through project SFRH/BD/62326/2009.

\section{References}

AS 5604-2005, 2005. Timber natural durability ratings. Australia: Standards Australia.

Brites, R. S., 2011. Safety assessment of ancient timber structures. PhD thesis, University of Minho.

Canada Mortgage and Housing Corporation, 1996. Survey of building envelope failures in the coastal climate of British Columbia. Canada Mortgage and Housing Corporation, Ottawa, ON, Canada.

Canada Mortgage and Housing Corporation, 2000. Wall moisture problems in Alberta dwellings. Canada Mortgage and Housing Corporation, Ottawa, ON, Canada.

Canada Mortgage and Housing Corporation, 2007. Wind-rain relationships in Southwestern British Columbia. Canada Mortgage and Housing Corporation, Ottawa, ON, Canada.

Chui, Y., Ni, C., 1998. Stress-strain relationship of common wire nails under reversed cyclic loading. Journal of Testing and Evaluation 26(5), 420-425. 
Clausen, C. A., Highley, T. L., Lindner, D. L., 2006. Early detection and progression of decay in L-joints and lap-joints in a moderate decay hazard zone. Forest Products Journal 56, 100-106.

EN 1991:1-3, 2003. Eurocode 1: Actions on Structures - Part 1-3: General Actions Snow Loads. CEN.

EN 1995:1-1, 2004. Eurocode 5: Design of Timber Structures. Part 1-1: General Common rules and rules for buildings. CEN.

EN 330, 1993. Wood preservatives. Field test method for determining the relative protective effectiveness of a wood preservative for use under a coating and exposed out-of-ground contact. L-joint method. CEN.

ENV 12037, 1996. Wood preservatives. Field test method for determining the relative effectiveness of a wood preservative exposed out of ground contact. Horizontal lapjoint method. CEN.

Foliente, G. C., Leicester, R. H., Wang, C.-H., Mackenzie, C. E., Cole, I. S., 2002. Durability design for wood construction. Forest Products Journal 52, 10-19.

ISO 13823, 2008. General Principles on the Design of Structures for Durability. Reference Number 13823:2008(E). Switzerland. ISO.

JCSS, 2001. Probabilistic Model Code. Joint Committee of Structural Safety. www.jcss.ethz.ch.

Kent, S., Leichti, R., Rosowsky, D., Morrell, J., 2005. Effects of decay on the cyclic properties of nailed connections. Journal of Materials in Civil Engineering ASCE $17,579-585$.

Köhler, J., Sørensen, J., Faber, M., 2007. Probabilistic modelling of timber structures. Structural Safety 29, 255-267.

Leicester, R. H., 2001. Engineered durability for timber construction. Progress in Structural Engineering and Materials 3, 216-227. 
Leicester, R. H., Wang, C.-H., Nguyen, M. N., Mackenzie, C. E., 2009. Design of exposed timber structures. Australian Journal of Structural Engineering 9, 217-224.

Ranta-Maunus, A., 2004. Theoretical and practical aspects of the reliability analysis of timber structures. In: WCTE 2004 - 8th World Conference on Timber Engineering.

Sawata, K., Sasaki, T., Doi, S., Iijima, Y., 2008. Effect of decay on shear performance of dowel-type timber joints. Journal of Wood Science 54, 356-361.

Shooman, M., 1968. Probabilistic Reliability: An Engineering Approach. McGrawHill.

Viitanen, H., 1994. Factors affecting the development of biodeterioration in wooden constructions. Materials and Structures 27, 483-493.

Wang, C., Leicester, R. H., 2008. A probabilistic procedure for design of timber aboveground under attack of decay fungi. In: Australian Structural Engineering Conference (ASEC 2008), Melbourne.

Wang, C., Leicester, R. H., Nguyen, M., 2008. Probabilistic procedure for design of untreated timber poles in-ground under atack of decay fungi. Reliability Engineering and System Safety 93, 476-481.

Zabel, R. A., Morrell, J. J., 1992. Wood Microbiology: Decay and its Prevention. New York Academic Press. 


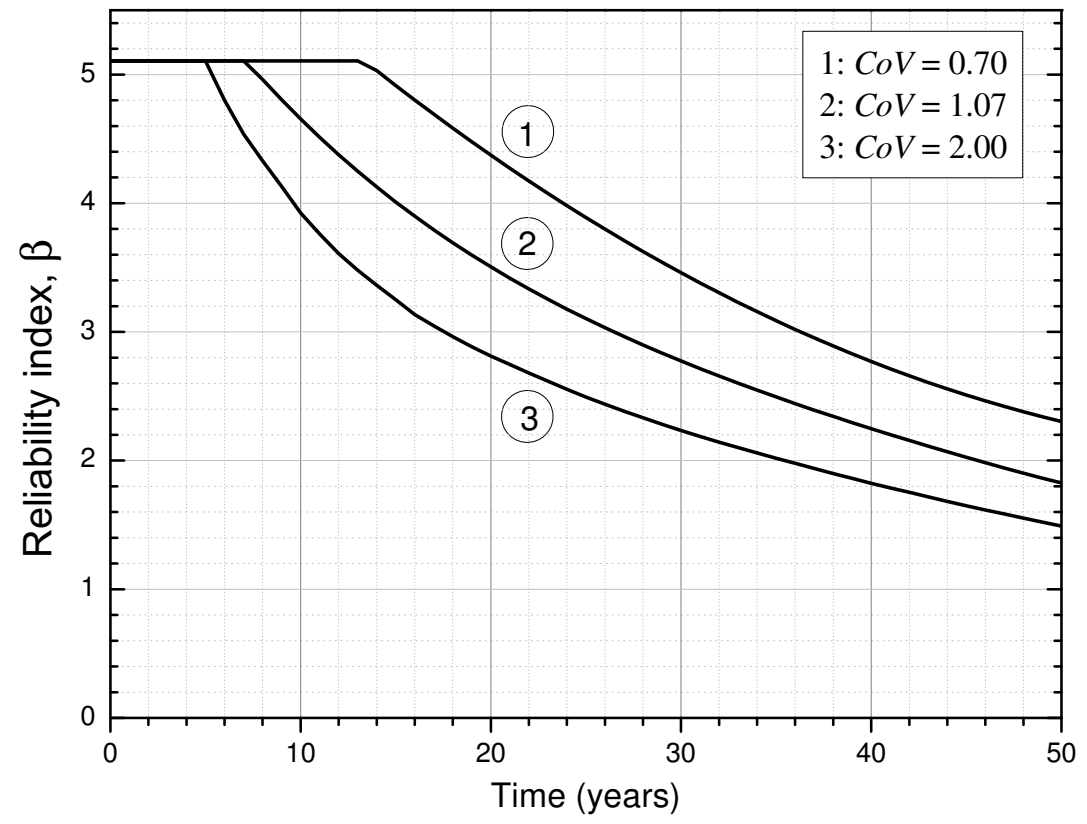

(a)

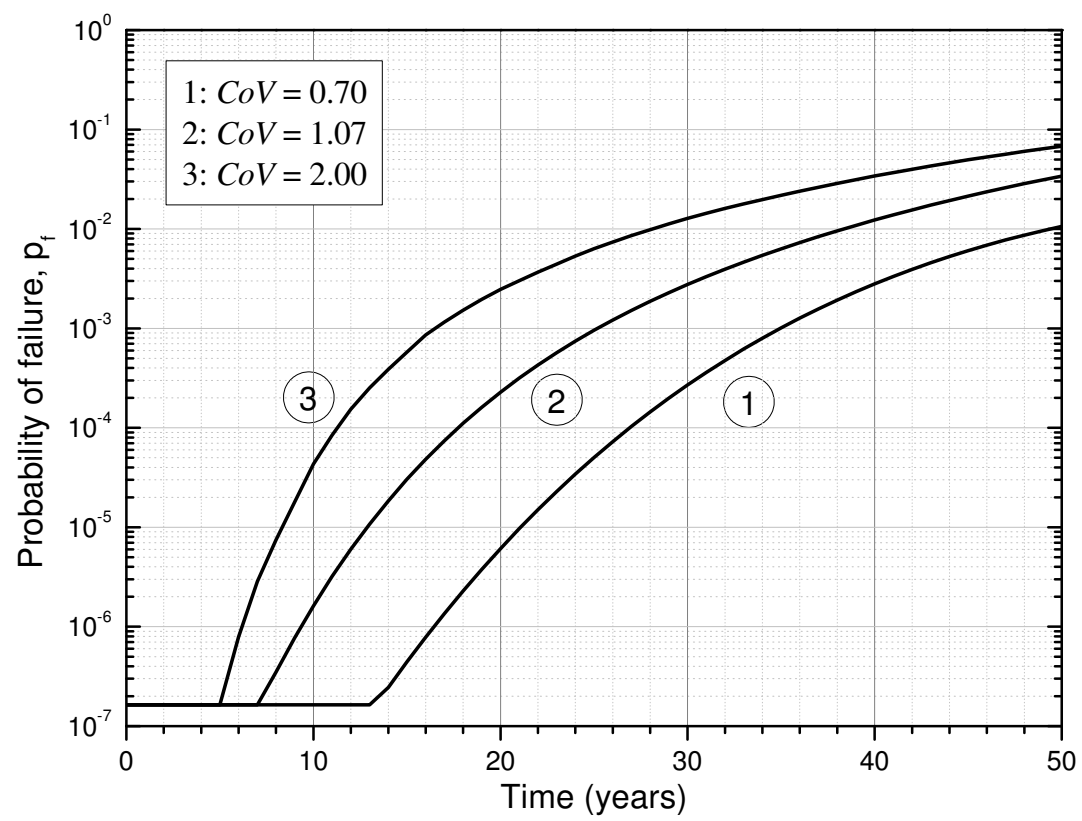

(b)

Figure 6: Evolution of (a) the reliability index and (b) probability of failure with decay for the entire structure, for a period of 50 years 


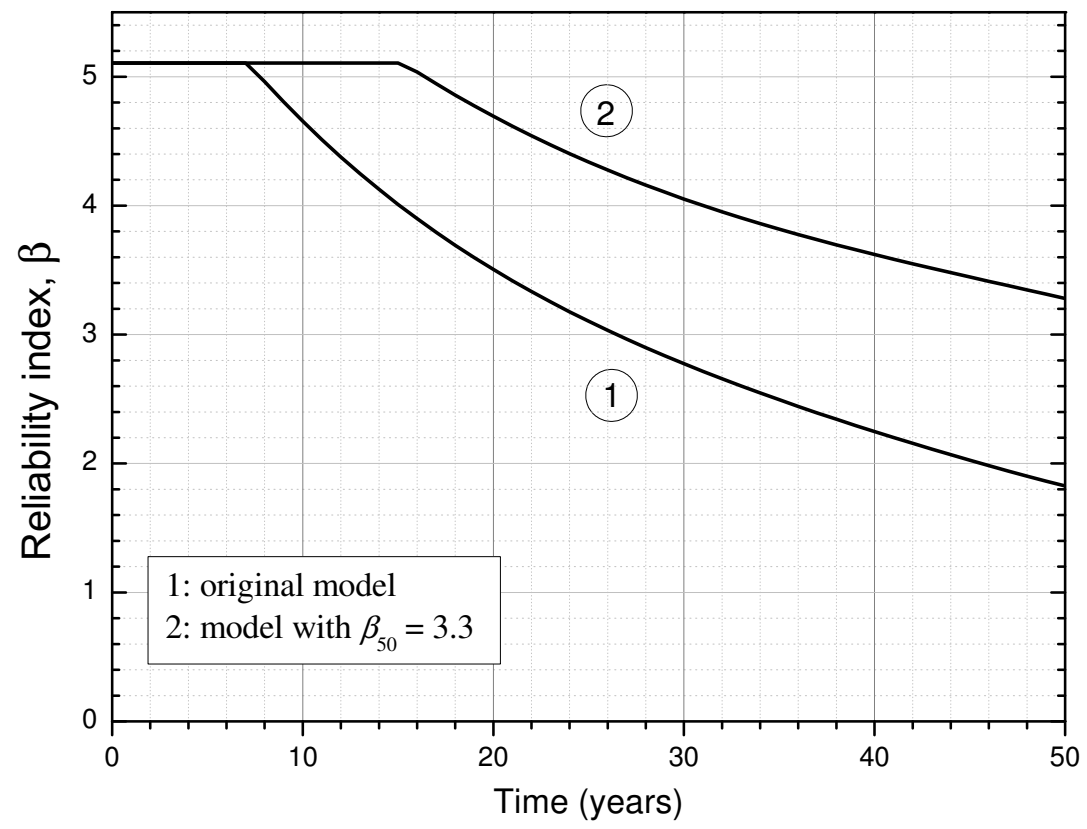

Figure 7: Evolution of the reliability index for the model calibrated to a target reliability level of $\beta_{\text {target }}=3.3$ after 50 years. 